\title{
Weinberg Energy-Momentum Complex for a Stringy Black Hole Solution
}

\author{
Irina Radinschi *and Brindusa Ciobanu ${ }^{\dagger}$ \\ Department of Physics, "Gh. Asachi" Technical University, \\ Iasi, 700050, Romania
}

\begin{abstract}
In our paper we compute the energy distribution of a magnetic stringy black hole solution in the Weinberg prescription. The metric under consideration describes the dual solution in the string frame that is known as the magnetic stringy black hole solution. The metric is obtained by multiplying the electric metric in the Einstein frame by a factor $e^{-2 \Phi}$. The energy distribution depends on the mass $M$ and charge $Q$. Also, we make a discussion of the results and we compare our result with those obtained in the Einstein and Landau and Lifshitz prescriptions and investigate the connections between the expressions of the energy obtained in these prescriptions.
\end{abstract}

Keywords: Weinberg energy-momentum complex, magnetic stringy black hole

PACS: 04. $20 \mathrm{Dw}, 04.70$. Bw

\section{INTRODUCTION}

The important issue of energy localization still lacks of an acceptable answer and continues to be one of the most interesting and challenging problem of the General Relativity. We point out that this problem of defining in an acceptable manner the energy-momentum density hasn't got a generally

*radinschi@yahoo.com

†bciobanu2003@yahoo.com 
accepted answer yet. The center of general relativity is one of the most interesting and challenging ideas in modern science, the one that gravity is the geometry of curved four-dimensional space-time. Mass is the source of the space-time curvature, and since General Relativity incorporates Special Relativity, any form of energy is also a source of space-time curvature. Also, the subject of the localization of energy continues to be an open one since Einstein [1] has given his important result of the special theory of relativity that mass is equivalent to energy.

Over the last two decades the issue of the energy-momentum localization by using the energy-momentum complexes was re-opened and many interesting results were obtained. In 1990 Bondi [2] gave his opinion that "a nonlocalizable form of energy is not admissible in general relativity, because any form of energy contributes to gravitation and so its location can in principle be found". Misner et al [3] sustained that to look for a local energymomentum means that is looking for the right answer to the wrong question. Also, they concluded that the energy is localizable only for spherical systems. On the other hand, Cooperstock and Sarracino [4] demonstrated that if the energy is localizable in spherical systems then it is also localizable in any space-times. The localization of energy is connected to the use of various energy-momentum complexes, including those of Einstein [5], Landau and Lifshitz [6], Papapetrou [7], Bergmann [8] and Weinberg [9]. All of these prescriptions have a drawback: the calculations are restricted to quasi-Cartesian coordinates. On the other hand, Møller [10] constructed an expression which enables one to calculate the energy distribution in any coordinate system not only in quasi-Cartesian coordinates. Also, in this context, of great importance is the Cooperstock [11] hypothesis which states that the energy and momentum are confined to the regions of non-vanishing energy-momentum tensor of the matter and all non-gravitational fields.

As we emphasized above, the problem of energy-momentum localization by using the energy-momentum complexes was revived at the begining of the last decade. The interesting results recently obtained by many researchers point out that the energy-momentum complexes are powerful tools for evaluating the energy and momentum in a given space-time [12]. Recently, important works were done with the energy-momentum complexes in 2- and 3-dimensional space-times [13]. All these considerations demonstrate the significance of these prescriptions and stress the usefulness of energy-momentum complexes for the localization of energy.

In our paper we compute the energy distribution of a magnetic stringy 
black hole solution in the Weinberg prescription. The metric under consideration describes the dual solution in the string frame that is known as the magnetic stringy black hole solution. The metric is obtained by multiplying the electric metric in the Einstein frame by a factor $e^{-2 \Phi}$. Also, we make a discussion of the results and we compare our result with those obtained in the Einstein and Landau and Lifshitz prescriptions and investigate the connections between the expressions of the energy obtained in these prescriptions. Through the paper we use geometrized units $(G=1, c=1)$ and follow the convention that Latin indices run from 0 to 3.

\section{ENERGY IN THE WEINBERG PRESCRIP- TION}

The study of the stringy black holes is an interesting issue because string theory is expected to provide us with a finite and clearly defined theory of quantum gravity and the answer of many questions related with black hole evaporation could be solved in the context of string theory. String theory may be the best way to attain the holy grail of fundamental physics, which is to generate all matter and forces of nature from one basic building block. Through the years, many important studies have been made related to the string theory. About the low energy effective theory we point out that largely resembles general relativity with some new "matter" fields as the dilaton, axion etc [14]-[15]. One of its main property is that there are two different frames in which the features of the space-time may look very different. These two frames are the Einstein frame and the string frame and they are related to each other by a conformal transformation $\left(g_{\mu \nu}^{E}=e^{-2 \Phi} g_{\mu \nu}^{S}\right)$ which involves the massless dilaton field as the conformal factor. The string "sees" the string metric. Many of the important symmetries of string theory also rely of the string frame or the Einstein frame [16]. The $T$ duality transformation relates metrics in the string frame only, whereas $S$ duality is a valid symmetry only if the equations are written in the Einstein frame. Kar [14] gave important results about the stringy black holes and energy conditions. He studied several black holes in two and four dimensions with regard to the Weak Energy Conditions (WEC).

The action for the Einstein-dilaton-Maxwell theory is given by 


$$
S_{E D M}=\int d^{4} x \sqrt{-g} e^{-2 \Phi}\left[R+4 g_{\mu \nu} \nabla^{\mu} \Phi \nabla^{\nu} \Phi-\frac{1}{2} g^{\mu \lambda} g^{\nu \rho} F_{\mu \nu} F_{\lambda \rho}\right] .
$$

Varying with respect to the metric, dilaton and Maxwell fields we get the field equations for the theory given as

$$
\begin{gathered}
R_{\mu \nu}=-2 \nabla_{\mu} \Phi \nabla_{\nu} \Phi+2 F_{\mu \lambda} F_{\nu}^{\lambda}, \\
\nabla^{\nu}\left(e^{-2 \Phi} F_{\mu \nu}\right)=0 \\
4 \nabla^{2} \Phi-4(\nabla \Phi)^{2}+R-F^{2}=0 .
\end{gathered}
$$

The metric (in the string frame) which solve the Einstein-dilaton-Maxwell field equations to yield the electric black hole is given by

$$
d s^{2}=A\left(1+\frac{2 M \sinh ^{2} \alpha}{r}\right)^{-2} d t^{2}-\frac{1}{A} d r^{2}-r^{2} d \theta^{2}-r^{2} \sin ^{2} \theta d \varphi^{2} .
$$

where $A=1-\frac{2 M}{r}$.

In the string frame the dual solution known as the magnetic black hole is obtained by multiplying the electric metric in the Einstein frame by a factor $e^{-2 \Phi}$. Therefore, the magnetic black hole metric is given by

$$
d s^{2}=\frac{A}{B} d t^{2}-\frac{1}{A B} d r^{2}-r^{2} d \theta^{2}-r^{2} \sin ^{2} \theta d \varphi^{2},
$$

with $B=1-\cdot \frac{Q^{2}}{M r}$, where $M$ is the mass and $Q$ is the charge of the magnetic black hole.

The Weinberg energy-momentum complex [9] is given by

$$
W^{i k}=\frac{1}{16 \pi} D_{, l}^{l i k}
$$

where

$$
D^{l i k}=\frac{\partial h_{a}^{a}}{\partial x_{l}} \eta^{i k}-\frac{\partial h_{a}^{a}}{\partial x_{i}} \eta^{l k}-\frac{\partial h^{a l}}{\partial x^{a}} \eta^{i k}+\frac{\partial h^{a i}}{\partial x^{a}} \eta^{l k}+\frac{\partial h^{l k}}{\partial x_{i}}-\frac{\partial h^{i k}}{\partial x_{l}}
$$

with 


$$
h_{i k}=g_{i k}-\eta_{i k}
$$

and $W^{00}$ and $W^{\alpha 0}$ are the energy and, respectively, the momentum density components.

The Weinberg energy-momentum complex satisfies the local conservation laws

$$
\frac{\partial W^{i k}}{\partial x^{k}}=0
$$

Integrating $W^{i k}$ over the three-space gives the energy and momentum components

$$
P^{i}=\iiint W^{i 0} d x^{1} d x^{2} d x^{3} .
$$

$P^{0}$ is the energy and $P^{\alpha}$ are the momentum components.

Using the Gauss theorem we obtain

$$
P^{i}=\frac{1}{16 \pi} \iint D^{\alpha 0 i} n_{\alpha} d S
$$

where $n_{\alpha}=\left(\frac{x}{r}, \frac{y}{r}, \frac{z}{r}\right)$ are the components of a normal vector over an infinitesimal surface element $d S=r^{2} \sin \theta d \theta d \varphi$.

For making the calculations using the Weinberg energy-momentum complex we transform the metric given by (6) to quasi-Cartesian coordinates $t, x, y, z$ according to $x=r \sin \theta \cos \varphi, y=r \sin \theta \sin \varphi$ and $z=r \cos \theta$.

Using (7), (8) and applying the Gauss theorem we obtain that the energy distribution of the magnetic black hole is given by

$$
E_{W}=\frac{r}{2} \frac{r Q^{2}+2 M^{2} r-2 M Q^{2}}{(r-2 M)\left(M r-Q^{2}\right)} .
$$

The energy distribution depends on the mass $M$ and charge $Q$ of the magnetic black hole.

We make a comparison with the values of the energy distribution obtained in the Einstein and Landau and Lifshitz prescriptions. The energy in the Einstein prescription [17] is given by

$$
E_{E}=\frac{1}{2} \frac{r Q^{2}+2 M^{2} r-2 M Q^{2}}{\left(M r-Q^{2}\right)}
$$


We establish the connection between the expressions of the energy distribution in the Weinberg and Einstein prescriptions that is

$$
E_{W}=\frac{r}{(r-2 M)} E_{E} .
$$

The expression of energy in the Landau and Lifshitz prescription is the same as in the Weinberg prescription. We conclude that between the Weinberg, Einstein and Landau and Lifshitz prescriptions there is a relationship given by

$$
E_{W}=E_{L L}=\frac{r}{(r-2 M)} E_{E}
$$

These definitions do not provide the same result for the energy distribution. It is important that the expression of the energy obtained in the Weinberg prescription exactly matches with that computed in the context of the Landau and Lifshitz prescription.

For the Weinberg and Landau and Lifshitz prescriptions we take the limit $Q \rightarrow 0$, the Schwarzschild space-time, and we obtain for the energy distribution the expression

$$
E_{W}=E_{L L}=\frac{M}{\left(1-\frac{2 M}{r}\right)}=M\left(1-\frac{2 M}{r}\right)^{-1} .
$$

This expression is the same as obtained by Virbhadra [18] in the case of the Schwarzschild black hole when the calculations were done in Shwarzschild Cartesian coordinates.

In the limit $r \rightarrow \infty$ we obtain for the energy distribution of the magnetic black hole

$$
\underset{r \rightarrow \infty}{E_{W}}=\underset{r \rightarrow \infty}{E_{L L}}=M+\frac{Q^{2}}{2 M}
$$

\section{DISCUSSION}

The subject of the localization of energy continues to be one of the most interesting and challenging problems of the General Relativity. This issue is an open one since Einstein has given his important result of the special theory of relativity that mass is equivalent to energy. Also, Chang, Nester and Chen [19] showed that the energy-momentum complexes are actually quasilocal 
and legitimate expression for the energy-momentum. They concluded that there exist a direct relationship between energy-momentum complexes and quasilocal expressions because every energy-momentum complexes is associated with a legitimate Hamiltonian boundary term. The concept of energymomentum complexes was reconsider by Virbhadra and his collaborators, and since then, many interesting results was obtained in this area [12]-[13]. Although, the energy-momentum complexes of Einstein, Landau and Lifshitz, Papapetrou, Bergmann and Weinberg are coordinate dependent they can give a reasonable result if calculations are carried out in quasi-Cartesian coordinates.

In this paper we evaluate the energy distribution of a magnetic stringy black hole solution in the Weinberg prescription. The energy distribution depends on the mass $M$ and charge $Q$ of the magnetic black hole. We compare this result with those obtained in the Einstein and Landau and Lifshitz prescriptions. These definitions do not provide the same result for the energy of the magnetic stringy black hole, particularly we point out that the Weinberg and Landau and Lifshitz prescriptions furnish the same expression for the energy distribution. The connection between the expressions of the energy distribution obtained in these three prescription is given by the relation $E_{W}=E_{L L}=\frac{r}{(r-2 M)} E_{E}$.

These results sustain that the energy-momentum complexes are powerful tools for obtaining the energy distribution in a given space-time.

\section{References}

[1] A. Einstein, Preuss. Akad. Wiss. Berlin 47, 778 (1915); Addendum-ibid. 47, 799 (1915).

[2] H. Bondi, Proc. R. Soc. London A427, 249 (1990).

[3] C. W. Misner, K. S. Thorne and J. A. Wheeler, Gravitation, W. H. Freeman and Co., NY, 603 (1973).

[4] F. I. Cooperstock and R. S. Sarracino, J. Phys. A: Math. Gen. 11, 877 (1978).

[5] R. P. Wallner, Acta Physica Austriaca 52, 121 (1980). 
[6] L. D. Landau and E. M. Lifshitz, The Classical Theory of Fields (Pergamon Press, 1987, p. 280).

[7] A. Papapetrou, Proc. R. Irish. Acad. A52, 11 (1948).

[8] P. G. Bergmann and R. Thompson, Phys. Rev. 89, 400 (1953).

[9] S. Weinberg, Gravitation and Cosmology: Principles and Applications of General Theory of Relativity (John Wiley and Sons, Inc., New York, 1972 , p. 165).

[10] C. Møller, Ann. Phys. (NY) 4, 347 (1958).

[11] F. I. Cooperstock, Found Phys. 22, 1011 (1992); Annals Phys. 282115 (2000); Mod. Phys. Lett. A14, 1531 (1999).

[12] K. S. Virbhadra, Phys. Rev. D41, 1086 (1990); S. Virbhadra, Phys. Rev. D42, 2919 (1990); N. Rosen and K. S. Virbhadra, Gen. Rel. Grav. 25, 429 (1993); J. M. Aguirregabiria, A. Chamorro and K. S. Virbhadra, Gen. Rel. Grav. 28, 1393 (1996); K. S. Virbhadra, Phys. Rev. D60, 104041 (1999); S. S. Xulu, Int. J. Theor. Phys. 37, 1773 (1998); S. S. Xulu, Int. J. Mod. Phys. D7, 773 (1998); S. S. Xulu, Mod. Phys. Lett. A15, 1511 (2000); S.S. Xulu, Astrophys. Space. Sci. 23, (2003); I. Radinschi, Acta Physica Slovaca, 49(5), 789 (1999); I. Radinschi, APH N.S., Heavy Ion Physics 12, 47 (2000); I. Radinschi, Mod. Phys. Lett. A15, Nos. 11\&12, 803 (2000); I. Radinschi, Chin. J. Phys. 39(3), 231 (2001); I. Radinschi, Chin. J. Phys. 39(5), 1 (2001); I. Radinschi, Mod. Phys. Lett. A16(10), 673 (2001); I-Ching Yang and I. Radinschi, Chin. J. Phys. 41, 326 (2003); I. Radinschi and I-Ching Yang, , New Developments in String Theory Research, Ed. Susan A. Greece, Nova Science Publishers, Inc. New York, U.S.A, 1-17, 2006, ISBN: 1-59454-488-3; I. Radinschi and Th. Grammenos, Int. J. Mod. Phys. A21, 2853 (2006); I-Ching Yang, Cheng-Chang Jeng, gr-qc/0605145, I-Ching Yang, ChiaHsiu Tsai, gr-qc/0503118; O. Patashnick, Int. J. Mod. Phys. D14, 1607 (2005); T. Bringley, Mod. Phys. Lett. A17, 157 (2002); M. Súkeník and J. Sima, gr-qc/0101026; M. Sharif and Tasnim Fatima, Int. J. Mod. Phys. A20, 4309 (2005); M. Sharif, Nuovo Cim. B19, 463 (2004); M. Sharif, Int. J. Mod. Phys. D13, 1019 (2004); R. Gad, Mod. Phys. Lett. A19, 1847 (2004); R. Gad, Astrophys. Space. Sci. 295, 451 (2005); R. Gad, Astrophys. Space. Sci. 293, 453 (2004); M. Salti, Nuovo Cim. 
120B, 53 (2005); M. Salti, Astrophys. Space. Sci. 299, 159 (2005); M. Salti and A. Havare, Int. J. Mod. Phys. A20, 2169 (2005); O. Aydogdu, Fortsch. Phys. 54, 246 (2006); O. Aydogdu and M. Salti, Acta Phys. Slov. 55, 537, (2005); P. Halpern, gr-qc/0606095; Sezgin Aygun, Melis Aygun, Ismail Tarhan, $g r-q c / 0607115 ; g r-q c / 0607110$; Figen Binbay, Irfan Acikoz, M. Salti, gr-qc/0607083; Nurettin Pirinccioglu, Irfan Acikoz, M. Salti, gr-qc/0607082

[13] E. C. Vagenas, Int. J. Mod. Phys. A18, 5949 (2003); E. C. Vagenas, Int. J. Mod. Phys. A18, 5781 (2003); E. C. Vagenas, Int. J. Mod. Phys. D14, 573 (2005); Th. Grammenos, Mod. Phys. Lett. A20, 1741 (2005).

[14] S. Kar, Phys. Rev. D55, 4872 (1997).

[15] M. S. Green, J. H. Schwarz and E. Witten, Superstring Theory, (Cambridge University Press 1987).

[16] A. Giveon, M. Porrati and E. Rabinovici, Phys. Rep.244, 177 (1994); A. Sen, Int. J. Mod. Phys. 9, 3707 (1994).

[17] I. Radinschi, Romanian Journal of Physics, Vol.50, Number 1-2 (2005).

[18] K. S. Virbhadra, Phys. Rev. D60, 104041 (1999).

[19] Chia-Chen Chang, J. M. Nester and Chiang-Mei Chen, Phys. Rev. Lett. 83, 1897 (1999). 